versalen geistigen Einfluß in der Wissenschaft. den sich die Mathematik durch ihre innerliche Vertiefung und ihre grobzingige Ausgestaltung erworben hatte, mit Nachdruck und Erfolg zur Geltung gebracht hat, indem er eine weitherzige philosophische Auffassung von der Mathematik entwickelte, welche es ermöglicht, der Bedeutung und 'Tragweite ilhrer Methode gerecht zu werden. Die Freunde der mathematischen Wissenschaft werden ihm dafür dauernden Dank wissen.

\title{
Verzeichnis der bisherigen Publikationen von David Hilbert
}

\author{
(nebst kurzen Inbaltsangabea).
}

Von Krarl Bifgel, Föttingen.

1. Uber die invaritulen Hifenschatten specielley binärer lormen, insbesondere der Kugelfunotionen; Inaugural-Dissertation (Königsberg i. Pr. 1885, R. Leupold).

Ein Verfahren zur Darstellung von Invarianten und Covarianten eines Systems binärer Formen wird allgemein begruadet und auf spezielle binäre Formen angewendet.

2. Uber cine allgemeine Gattung irrationaler Invarianten und Covarianten fïr eine binäre Grundform geraden Grades. Berichte iiber die Verhandlungen der Königlichen Sächsischen Gesellschait dor Wissenschaften zu Leipzig, mathematisch-phy. sische Classe, Bd. gr (1885), S. 427-438.

Zur Erforschung der analytischen Natur und Bedeutung der invarianten Bildungen werden irrationale Invarianten eines Systems von Grundformen untersucht.

3. Uber die nothucndigen und hinreichenden covarianten Bedingungen für die Darstellbarkeit einer binaren Form als vollständiger Potenz. Mathematische Annalen Bd. 27 (1886), S. 158-161.

Die notwendige und hinreichende Bedingung wird durch das identisehe Versehwinden einer gewissen Covariante geliefert.

4. Uber einen allgemeinen Gesichtspunkt für invariantentheoretische Untersuchwngen in binarent Formengebiete (Königsberger Habilitationsschrift). Nathematische Annalen Bd. 28 (1887), S. 381-446.

Weitertührung der vorläufigen Mitteilung 2.

5. Über eine Darstellungsueise der invarianten Gebilde im binärn Formengebiete. Mathematische Annalen B.d. 30 (1887), S. 15-29.

Verkiurzte Wiedergabe der Dissertation 1.

i. Uber die Singularitäten der Discriminantenfläche. Mathematische Annalen Bd. 30 (1887), S. 437-441.

Bestimmung der Gesamtordnung der singulären Gebilde der Discriminantenfläche.

7. Uber binäre Formenbïschel mit besonderer Combinanteneigenschaft. Mathematische Annalen Bd. 30 (1887), S. 561-570.

Ablutung einiger sätze uber das identisehe Verschwinden von Uberschiebungen zweier Formon.

6. Uber dic Bïschet von binüren Formen mit dcr sämlichen Functionaldeterminanto. Berichte uber die Verhandlungen der Königlichen Sächsischen Gesellschaft der Wissenschaften zu Leipzig, mathematisch-physische Clasee, Bd. 39 (1887), S. 112 bis 122 .

Aufistellung aller Formenbüschel von gegebener Funktionaldeterminante. Vgl. auch 15.

9. Uber binäre Formen mit vorgeschriebener Discriminante. Mathematische Annalen Bd. 31 (1888). S. $482-492$.

Alle Formenbüschel $x \varphi+\mu \psi$ werden be- stimmt, deren biscriminante eine gegebene binäre Form 2ter, 4ter, bter Ordnung der Variabeln $\%$, ist.

11. Uber dio Discriminante der in Endlichen abbrechenden hypergeonetrischen Reihe. Journal fïr die reine und angewandte Mathematik $\mathrm{Bd} .103$ (1888), S. $337-345$.

Neue Ableitung des von stieltjes gegebenen Ausdrucks der Diseriminante.

11. Let e adressée à $M$. Hermite. Journal de Mathématiques pures et appliquées, 4. Reihe, Bd. 4 (1888), s. $249-256$.

Anwendung eines allgemeinen Prinzips auf die Untersuohung biquadratischer binärer und kubischer ternärer Formen.

12. Uber die Darstellung definiter Formen als Summe von Formenquadraten. Mathematisehe Annalen Bd. 32 (1888), S. 342-350.

Beweis der Vermutung von Minkowski über die Existenz definiter Formen gerader Ordnung, welche nicht als Summe von Quadraten endlick vieler reeller Formen darstellbar sind.

13. Zur Theorie der algobraischen Gebilde. Nachrichten von der Königliwen Gesellschaft der Wissenschaften zu Göttingen, mathematisch-physikalische Klasse, Jahrgang 1888, S. 450-457.

Vgl. 18.

14. Uber die Endlichkeit des Invariantensystems für binäre Gundformen. Mathematische Annalen Bd. 33 (1889), S. 223-226.

Neuer Beweis des Satzes von Gordan iiber die Endlichkeit des Invariantensystems.

15. Uber Büschel von binären Formen mit vorgeschriebener Functionaldeterminante. Mathematische Annalen Bd. 39 (1889). S. 227-236.

Vgl. 8.

16. Zur Theorie der algebraischen Gebilde II. Nachrichten von der Königlichen Gesellschaft der Wissenschaften zu Göttingen, mathematisch-physikalischo Klasse, Jahrgang 1889, S. 25-34.

Vgl. 18.

17. Zur Theoric der algebraischen Gebilde $I I I$. Nachrichten von der Koniglichen Gesellschaft der Wissenschaften zu Göttingen, mathematisch-physikalikche Klasse, Jahrgang 1889. S. 423-430.

$\mathrm{V} \mathrm{gl}, 18$.

18. Uber dio Theorie der algebraisohen Formen. Mathematisohe Annalen Bd. 36 (1890), S. 473-534.

Beweis der Satze: 1. Zu jeder Folge von Formen $F_{1}, F_{g}, \ldots$ in $n$ Variabeln gibt es eine Zahl $m$ denart, daß jede Form $F$ der Folge in der Gestalt

$$
F=A_{1} F_{1}+\cdots+A_{11} F_{\mathrm{Jn}}
$$

darstellbar ist, wo $A_{\mathrm{t}} \ldots, \ldots A_{\mathrm{m}}$ gewisse Formen derselben Variabeln bedeuten; haben dabei $F_{1}$, 
$F_{2}, \ldots$ ganzzahlige Koeffizienten, so gilt dasselbe von $A_{1}, \ldots, A_{\mathrm{m}}$. 2 . Bei jedem System von Grundformen beliebig vieler variabeln reihen, welche denselben oder verschiedenen Transformationen unterhegen, lassen sich alle ganzen rationalen Invarianten als ganze rationale Funktionen von endlich vielen festen ganzen rationalen Invarianten ausdrükcken. Vgl, auch 13, 16., 17.

19. Über die reellen Züge algebraischer Curven. Mathematische Annalen Bd. 38 (1891), S. 115-138.

Aufstellung aller gastaltlich verschiedenen Arten von Ramkurven fester Ordnung nit der Maximalzahl reeller Ziige.

20. Uber die stetige Abbildung einer Linie auf ein Flächenstuick. a) Verhandlungen der Gesellschaft deutsoher Naturforseher und Arzte, 63. Versammlung zu Bremen, 15.-20. September 1890 (Leipzig 1891 , F. C. W. Vogel), S. 11-12. b) Mathematische Annalen Bd. 38 (1891), S. $459-460$. c) Prace matematyczo-fizyczne Bd. 5 (1894), S. 13-14 (ins Polnische ïbersetzt ron S. Dickstein unter dem Titel: o odworowaniu ciagtem linit no kawalku powierzchni).

Angabe eines einfachen Beispiels für die Abbil. dung eines Quadrates auf eine Strecke.

21. Ubber die diophantischen Gleichungen vom Geschlecht Null. Acta mathematica Bd. 14 (1891), S. 217 -224. (Zusammen mit A. Hurwitz.)

Anwendüng der Sätze von $M$. Noether über birationale Transformation zur Rieduktion diophantiseher Gleichungen vom Grade $n>3$ und Geschlecht 0 auf solche zweiten oder dritten Grides.

22. Ober die Theorie der algebraischen Invarianten. Naehrichten von der Könighichen Gesellschaft der Wissenschaften $z u$ Göttingen, mathematisch-physikalische Klasse, Jahrgang 1891, S. 232-242.

V.gl. 27.

23. tber die Irreducibilitä ganzer rationaler Functionen mit yanzzahligen Coeffieienten. Journal fiir die reine und angewandte Mathematik $B d .110$ (1891), S. 104-129.

Beweis dres Satzes: Ist ein Polynon von $n(>2)$ Variabeln in einem algebraischen Zahlkörper irreduzibel, so lassen sich für irgend $*(<n)$ von den Variabeln solche ganzen ratio. nalen Zahlen einsetzen, daB das neue Polynom von $n-r$ Variabeln auch noch irreduzibel ist.

24. Uber volle Imvariantensysteme. a) Verbandlungen der Gasellschat deutscher Naturforscher, und Xrzte, 64. Versammlung zu Halle a. S. 21. bis 25. September 1891 (Leipzig 1891/92, F. C. W. Vogel), S. 11-12. b) Jahresbericht der Deutschen Mathematiker-Vereinigung, Bd, 1 (1892), S. 61-62.

Vigl. 27.

25. Ober die Theorie der algebraischen Invarianten $I I$. Nachrichten von der Königlichen Gesellsehaft der Wissenschaften zu Göttingen, mathematisch-phy* sikalische Klasse, Jahrgang 1892. S. 6-16.

Vigl. 27.

26. Vber dic Theorie der algebraischen Invarianten III. Nachrichten von der Königlichen Gesellschaft der Wissenschaften zu Göttingen, mathematisch. physikalische Klasse, Jahrgang 1892, S, 439-449.

Vgl. 27.

27. Dber die vollen Invariantensysteme. Mathematische Annalen Bil. 42 (1893), S. 313-373.

Unter Benutzung von 18. wird eine Methode zur Aufstellung des vollen Invariantensystems von Gruadformen beliebig vieler Variabeln begründet. Vgl auch 22., 24., 25, 26.

28. Uber ternäre definite Formen. Acta mathematica Bd. 17 (1893), S. $169-197$.

Beweis des Satzes: Jede ternäre definite Form ist Quotient zweier Summen yon Quadraten reeller Formen.

29. Viber die Transcendenz der Zahlen e und $\pi$ a) Nachrichten von der Königlichen Geselschaft. der Wissenschaften zu Göttingen, mathematischphysikalische Klasse, Jahrgang 1893, S. 113 bis 116. b) Mathematische Inualen Bd. 43 (1893), S. 216--219. c) Prace matematyczno-fizyczne, Bd. 5) (1894), S. 1-5 (ins Polnische uibersetzt ron S. Dickstein unter dem Titel: o pracstepnosici liczb $e i \pi$ ).

Vereinfachung der Beweise von Hermite und Lindemann.

30. Grundaüge einer Theorie des Galois'schen Zahlkörpers. Nachrichten von der Königlichen Gesell. schaft der Wissenschaften zu Göttingen, mathematisch-physikalische Klasen, Jahrgang 1894, S. $224-236$.

Füz jeden Galoisschen Körper verden gewisse besonders wiehtige Unterkörper (Zerlegungsköryer, Trägheitskörper, Verzweigungskörper) definiert.

31. Ein Beitrag zur Theorie des Legendre'schen Poly. noms. Acta mathematica Bd. 18 (1894), S. 155 bis 160 .

Berechnung der Discriminante der quadratischen Form

$$
\int_{0}^{1}\left(x_{1}+t x_{2}+\cdots+t^{n-1} x_{n}\right)^{2} d t
$$

mit Hilfe der Kugelfunktionen.

3:. Zwei neue Beweis: fir die Zerlegburkeit der Zahlen cines Körpers in Primideale. Jahres. bericht der Dentschen Mathematiker-Vereinigung Bu. $3(1894)$, S. 59.

$$
\text { Vol. } 33 .
$$

33. Uher die Zerlegung der Ideale eines Zahlenkörpers in Primideale. Mathematische Annalen $\mathrm{Bd}$. 4t (1894), S. $1-8$.

Der Satz von Dedekind uber die Eindeutigkeit der Primidealzerlogung wird unter Benutzung eines Galoisschen Körpers bewiesen.

Vgl. aluch 33 .

34. Uber den Dirichlet'schen biquadratischen Zahlkörper. Mathematische Annalen Bd. 45 (1894). S. $309-340$.

Rein arithmetische Begründung der Theorie derjenigen biquadratischen Zahikörper, welche dir Zahl $\sqrt{-1}$ enthalten; Einteilung seiner Ideal. klassen in Geschlechter; Ableitung des Dirichletschen Reziprozitätsgesetzes der quadratischen Reste im Körper der Gaubschen ganzen komplexen Zahlen.

35. Uber die gerade Linie als kürzeste Verbindung zueier Punkte a) Mathematische Annalen Bul if (1895), S. 91-96. b) L'enseignement' mathématiqne B. . 3 (1901). \&. 104--200 (tbersetzung ins Französische von $L$. Laugel unter dem Titel: Su lo ligne droite regardée comme étant le plus court chemin d'un point do un autre.

Axiomatisehe Begrindung des Satzes: In jedem Dreieck ist die Summe zweier Seiten nicht kleiner als die dritte Seite. 
36. De Seguier. Formes quadratiques et multiplication complexe d'après Kronecker. Göttingische gelehrte Anzeigen, Juhrgang, 1895, Bd. I, s.. 11-1.4. Besprechung des Buches von Seguier.

37. Ein neucr Beueis des Kronecker'schen Fundumentalsatzes über Abelsche Zahllorper. Nachrichten von der Königliohen Gesellschat der Wissenschaften zı Göttingen, mathematisch-physikalische Klasse, Jahrgang 1896, \$. 29-39.

Rein arithmetischer Beweis des satzes: Allo absolut $\Lambda$ belschen algebraiachen Zahlkörper sind Kroikkörper.

38. Zur Theorie der aus $n$ Haupteinhciton gebildeten complexen Größen. Nachrichten von der Königlichen Gesellschaft der Wissenschaften zu Göttingren, mathematisch-physikalische Klasse, Jahrgang 1896 , S. $179-183$.

Ableitung eines Satzes von Dodekind über hyperkomplexe Größen mit Hilfe eines in der Theorie der algebraischen Invarianten benutzten Satzes.

39. Lber die Theorie der algebraischen Invarianten. Mathematical Papers read at the International Mathematical Congress beld in connection with the world's Columbian Exposition Chicago 1893 (New York 1896, Macmillan and Co.), S. 116 bis 124

Utbersicht über die Entwieklung der Invariantentheorie.

40. Zum Gedächtnis an Karl Weierstraß. Nachrichten von der Königlichen Gesellschaft der Wissenschaften zu Göttingen, Geschäftliche Mitteilun. gen, Jahrgang 1897 , S. $60-69$.

41. Wie Theorie der algebraischen Zahlkörper. Jahresbericht der Deutschen Mathematiker-Vereinigung Bil. If (1897), S. I-XVIII und 175-546.

Bericht über die höhere Arithmetik mit kur zen Beweisen der Sätze; ausführliche Darstellung der Theorie des Kummerschen Zahlkörpers. Vgl. auch 68. und 76 .

42. Uber diophantische Gleichungen. Nachrichten von der Königlichen Gesellschaft der Wissenschaften zu Göttingen, mathematisch-physikalische Klasse, Jahruang 1897 , S, 48-54.

Beweis des Satzes: Die Diskriminante eines Polynoms vom Grade $\geq 4$ mit ganzen rationalen Koeffizienten ist von $\overline{ \pm} 1$ verschieden.

43. Über die Entuicklung einer beliebigen analytischen function einer Variabeln in cine unend7iche, nach ganzen rationalen Functionen fortschreitende Reihe. Nachrichten von der Königlichen Geselischaft der Wissensehaften zu Götingen, mathematisch-physikalische Klasse, Jahrgang 1897. S. $63-70$.

Verschärfung des Satzes von Runge uber die Entwicklung analytischer Funktionen in eine Reihe rationaler Funktionen.

44. Öber die Theorie der relativ-Abelschen Zahlkörper. Nachrichten von der Königlichen Geselschalt der Wissenschaften zu Göttingen, mathematisch-physikalische Klasse, Jahrgang 1898, S. 370-399.

Vigl. 54.

45. Uber die Theorie des relativquadratischen Zahlkörpers. Mathematische Annalen Bd. 51 (1899). S. $1-127$.

Beweis des alugemeinen Reziprozitätsgesetzes der quadratisehen Reste für total imaginäre Körpor mit ungerader Klassenzahl.
46. Uber die Theorie der relativquadratischen ZahlForper. Jahresbericht der Deutschen Mathematiker-Vureingoung Bd. 6 (1899), S. 88-94.

Verallemeinerung los quadratischen Rezipro. xitiitsgeisetzes anf beliebige Grundkörper.

47. Grundugen der Geometrie. al Festuchift zur Feier der Enthullung des GauB-Weber-Denkmalis in Göttingen (Teipzich 1899. B. G. Tenbner), 92 S.; 2. Aufl. (Leipzig 1903, B. G. Teubner). V + 175 S.; 3. Aull (Lipzig und Berlin 1909, B. G. Teubner), VI +279 S.; 4. Aufl. (Leipzig and Berlin 1913 B. r. Teubner). VI $+258 \mathrm{~S}$. b) Annales scientifiques de l'école normale sipérieure, 3. Reihe, Bd. $1 \%$ (1900), S. 103-209 (etwas veränderte thertragung ins Französiscle von L. Laugel unter dem Titel: Les principes fondamentunx do la géométrie). c) (Chicago 1902, The Open Court Publishing Company) VII +132 S. (ine Englische itbersetzt von $k$. J. Tounsend unter dem Titel: The foundations of geometwy).

Axiomatische Begrüdung der Geometrie; Beweis der Widerspruchslosigkeit; Untersuchung der Säze von Pascal und Desargues.

48. Mathematische Probleme. Vortrag, gehalten auf dem internationalen Mathematikerkongre $\beta$ zu Paris 1900. a) Nachrichten von der Königlichen Gesellschaft der Wiasenschaften zu Göttingen. mathematisch - physikalisehe Klasse, Jahrgang 1900, S. 253-297. b) Archiv der Mathematik und Physik, 3, Reihe, Bd. 1 (1900), S. $44-63$ und S. $213-237$. c) Lenseignement mathématique Bd. $2(1900)$, S. 349-355 (Auszug in französischer Ubersetzung unter dem Titel: Problèmes mathématiques). d) Compte rendu du deuxième congres international des mathematiciens tenu a Paris du 6 au 12 aont (Paris 1902, GauthierVillars), S. 58-114 (ins Französische ibersetzt von $L$. Laugel unter dem Titel: Sur les problèmes fulurs des mathématiques), e) Bulletin of the American Mathematical Society, 2. Reihe, Bd. 8 (1902), S. 437-479 (ins Englische ubersetzt von Mary Winston Nowson unter dem Titel: Mathematical problems).

49. Uber den Zahbegriff. a) Jahresbericht der Dentschen Mathematiker-Vereinigung Bd. 8 (1900), S. 180-184. b) (ins Russische übersetzt ron A. Wassilieffl.

Vortrag über die axiomatischen Grundlagen der Arithmetik.

50. Uber das Diriehlctsone Prinzip. a) Jahresbericht der Deutschen Mathematiker-Vereinigung Bd. 8 (1900), $8.184-188$. b) Nonvelles annales de mathématiques, 3. Reihe, Bd. $19(1900)$, S. 337 bis 344 (ine Französische uibersetzt von $L$. Laugel unter dem Titel: Sur le principe de Dirichlet). c) Journal für die reine und angewaydte Mathematik Bd. 129 (1905), S. $63-67$.

Findiexung der Methode des Dirichletsehen Prinzips; Anwendung zur Lösung von Variationsproblemen.

51. Uber das Dirichletsche Prinaip. a) Festschrift zur Feier des 150jährigen Bestehens der Königlichen Gesellschaft der Wissenschaften zu Göttingen, $1901,27 \mathrm{~S}$. b) Mathematische Annalen Bd, 59 (1904), S. $161-186$.

Von 50. versehiedene Begrundung des Dirichletschen Prinzips, dargestellt am Existenzbeweis der Integrale erster Gattung auf einer gegebenen Riemannschen Fläche. 
52. Uber Flächen von konstanter Gaußscher Krümmung. Transactions of the American Mathematical Society, B.d. 2 (1902), S. 87-99.

Beweis der Sätze: 1. Es gibt keine singularitäteufreie analytische Fläche von konstanter negativer Krümmung. 2. Die Kugel ist die einzige im Endlichen geschlossene singularitatenfreie analytische Fläche von konstanter positiver Krümmung.

53. Über die Grundlagen der Geometrie. a) Nachrichten von der Königlichen Gesellschaft der Wissenschaften zu Göttingen, mathematischphysikalische Klasse, Jabrgang 1902, S. 233-241. b) Mathematische Annalen Bd. 56 (1902), S. 381 bils 422 .

Axiomatische Begründung der Euklidischen und Bolyai-Lobatschefskijschen Geometrie.

54. Uber die Theorie der relativ-Abelschen Zahlköpper. Acta mathematica Bd. 26 (1902), S. 99-131.

Aufstellung der wichtigsten Satzo uber relativquadratische Zahlkörper bei beliebigem algebraiwhen Grundkörper und über den Klassenkörper eines algebratschen Zahlkörpers. Vgl. aueh 44.

55. Neue Begrïndung der Bolyai-Lobatschefskyschen Gcometrie. Mathematische Annalen Bd. $5^{\text {r }}$ (1903), S. $137-150$.

Die Geometrie von Bolyai-Lobatschefskij wirl ohne Stetigkeitsaxiome begründet.

56. Uber den Satz von der Gleiohheit der Basisuinkel im gleichschenkligen Dreieck. Procedings of the London Mathematical Society Bd. 35 (1903), S. $50-68$.

Untersuchungen ïber die zur ITerleitung des Satzes notwendigen Axiome.

57. Theorie der algebraischen Zahllörper. Enzyklopädie der mathematischen Wissenschaften mit Finschlub ihrer Anwendungen Br. I \& (Teipzig 1900-1904. B. G. Teubner), S. 675-698. Vgl. auch 79 .

58. Theorie der Kreiskörper. Ebentaselbst. S. 699 bis 714. Vgl. auch 79 .

59. Grundzüge cincr allgemeinen theorie dor linearen Integralglciohungen. Nachrichten von der Königlichen Gosellschatt, der Wissensehaften zu Cöttingen. mathematisch-physikalische Klasse. Erste Mitteilung, Jahrgang 1904. S. 49-91; 7weite Mitteilung. Jahroang 1904. S. 213-259; Dritte Mitteilung, Jahrgang 1905, S. 307-338; Vierte Mitteilung, Jahrơng 1906. S. 157-227; Fünfte Mitteilung. Jahroang 1906. S. 439-480; Sechste Mitteilung, Jahrgang 1910. S. 355-417.

Funktionen unendlich vieler Veränderlicher: Tifteare Integralgleichungen; Anwendungen auf grewöhnirgle und partielle Differentialgleichungen. Funktionentheorie. Variationsrechnong. Geo. metrie, Hyrlodymamik. Vgl, auch 69.

60. Ober die Grundlagen der Logik und der Arith. metik, a) Verhandlungen des dritten internatio. nalen Mathematiker-Kongresses in Heidelherg vom 8, bis 13. Augrist 1904 (Tripzi 1905 , B. C. Teubner). \$. 174-185. b) Lenseignement mathe matique Bu, $7 \quad(1905), S .89-103$ (ins Französische ibrersetzt unter dem Titel: Sur les fow dements de lin lorique et de larithmétique). c) The Monist, Jahrzang 1905. S. 338-352 (thes Fnglische ibersetst von a. B. Malsted unter dem Titel: On the foundations of logio and arithmetiol.

Vortrag iber die Widerspruchelosigkeit der Axiome der Arithmetik.
61. Über eine Amwendung der Integralgleichungen auf ein Problem der Funktionentheorie. Verhandlungen des dritten internationalen MathematikerKongresses in Heidelberg vom 8. bis 13. August 1904 (Leipzig 1905, B. G. Teubner), S. 233-240.

Existenzbeweis fiir eine in einem Gebiete analytische Funktion, deren Real- und Imaginäteil auf dem Rande einer linearen Relation genimgen.

62. Zur Variationsrchnung. a) Nachrichten von der Königlichen Gesellschaft der Wissensohaften zu röttingen, mathematisch-physikalisehe Klasse, Jahrgang 1905, S. $159-180$. b) Mathematisele Annalen Bd. 62 (1906), S. 351-370.

Ubertragung der Methode des unabhängigen Integrals auf Doppelintegrale.

63. Wesen und Ziele eincr Analysis der unendlichvielen unabhängigen Variabeln. Rendiconti del Circolo Matematico di Palermo Bd. $2 \gamma$ (1909), S. $59-74$.

Ableitung einiger Sätze über analytisele Funktionen mit unendlich vielen Veränderlichen.

64. Beueis für die Darstellbarkeit der ganzen Zahlen durch eine feste Anzahl nter Potenzen (Waringsahes Problem). Dem Andenken an IIermann Minkouski gewidmet. a) Nachrichten von der Konighichen Gesellschaft der Wissenschaften $z u$ Göttingen, Mathematisch-physikalische Klasse, Jahrgang 1909 , S. 17-36. b) Mathematische Annalen Bd. 67 (1909), S. $281-300$.

Bewein des Satzes von Waring iber die //erlegung natirlicher Zahlen in nte Potenzen.

(ii). ITermann Minkouski. Gedächtnisrede, gchaltew in der offontlichen Sitzung der Königlichen Gesollschaft der Wissenschaften $\approx u$ Göttingen am 1. Hai 1909. a) Nachrichten von der Königlichen Gesellschaft der Wissenschaften zu Göttingen. Geschäftiche Mitteilungen, Jahrgang 1909, \&. 72 bis 101 . b) Mathematisehe Annalen Bd. 68 (1910), 5. $445-471$.

60. Ober die Gestalt ciner flache vierter Ordnum. Nachrichten von dr. Königlichen Gesellschaft der Whssensehaften zu Gottingen, mathematisch-physikalische Klasse, Jahroang 1909, S. 308-313.

Nachweis der Fxistenz ciner singularitätenfreien Fïche vierter Ordnung vom Maximalrange 12 .

67. Zur Theoric der konformen Abbildang. Nachrichten von der Königlichen Gesellschaft der Wissensehaften zu Gottingen, mathematisch-plysikalische Klasse, Jahrgang 1909, S. 314-323.

Anfstellung cines Satzes iber die konformo slubildung eines beliebig oft zusammenhängenden beliebig vielblittrigen Cebietes anf einen Schlitzbereich.

18. Théorie des corps de nombres algébriques (Tradnit par M. A. Lévy, Professor au Tycé Voltaire). Annales de la Faculté des Sciences de l'Trniverziti: de Tonlouse, 3, Reihe, Bd, 1 (1909), S. 257 bia 328, und 3. Reihe, Bd. 2 (1912) S. 225-456.

Französische Ubersetzung von 41 .

(i0. Grundzüge einer allgemeincn Thorie der Tincaren Integralgleichungen. Nachrichten von der Königlichen Gesellschaft der Wissenschatten zn Göttingen, mathematisch-physikalische Klasse, Jahrgang 1910, S. $595-618$.

Sachlich geordnete Wiedergabe des Inhalto der 6 Mittelungen 59 , ohne Beweise. 


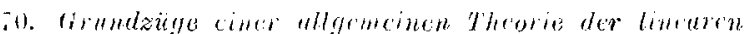
Integralgleidhayen. pleipzig und B(rlin 1!1:, 3. G. Teubwer), XXVI + 282 s.

Abdruck ron 59 , und 71 .

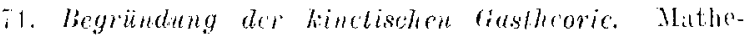
matische Annalen B1. it (1912), s. $562--577$.

Zurücktührung der Fundamuntalformel vou Maxwell-Bollamann in der kinetinchen Gastheorie auf eine lineare Integralgleichung zweiter Art. Vigl. auch 70 .

7.. Uber den Begriff der Klasse von Differentialglcichungen. il) Festschrift Heinrich Weber, zu seinem siebzigwten Geburtistag am 5. März 1912 gewidmet von Freumden und Schiilern (Leipzig und Berlin 1912, B. G. Teubner), S. 130-146. b) Mathematische Annalen Bd. 73 (1912), S. 95 bis 108.

7wei Differentialgleichungen mit zwei unabhängigen und einer abhängigen Variabeln nennt man zur selben Klasse gehörig, wenn sie umkehrbar integrallos ineinander transformiert werden können. Nachweis der Existenz mehrerer Klassen.

73. Begründung der elementaren Strahlungstheoric. a) Nachrichten von der Königlichen Gesellschaft der Wissenschaften zu Göttingen, mathematischphysikalische Klasse, Jahrgang 1912, S. 773-739. b) Physikalische Zeitschrift, 13. Jahrgang (1912), s. 1056-1064. c) Jahresbericht der Deutschen Mathematiker-Vereinigung. Bd. 22 (1913), S. 1-15.

Ableitung der Sätze von Kirchhoff mit Hilfe der Theorie der linearen Integralgleichungen.

74. Zusatz zur Begrïndung der elementaren Strahlungstheorie. Jahresbericht der Deutschen Mathematiker-Vereinigung Bd. 22 (1913), S. 16-20.

7). Bemerkungen zur Begründung der elementaren Strahlungstheorie. a) Nachrichten von der Königlichen Gesellschaft der Wissenschaften zu Götin. ren. Mathematisch-physikalische Klasse, Jahrgang 1913, S. 409-416. b) Physikalische Zeitschrift. 14. Jahrgang (1913), S. $592-595$.

Axiomatische Grundlagen der Sätze von Kirchhoff.

76. Théorie des corps de nombres algébriques (Traduitpar A. Lévy et Th. Got), (Paris 1913, A. Hermann et Fils), XVI + 380 S.

Französische Úbersetzung von 41, mit Zusätzen von Humbert und Got.

77. Zur Begrïndung der elementaren Strahlungstheorie (dritte Mitteilung). a) Nachrichten von der Königlichen Gesellschaft der Wissenschaften zu Göttingen, mathematisch-physikalische Klasse. Jahrgang 1914, S. 275-298. b) Physikalische Zeitschrift, 15. Jahrgang (1914), S. 878-889.

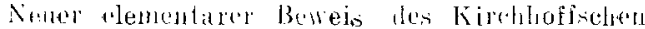
Siatam; Nachwes der Widerspruclislosigkeit drer benulztin Axiome.

is. Tlber dic lnvarianten cines systems eon beliebig

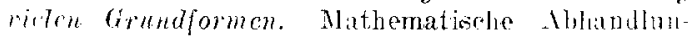
gen ITermann Amanlus Soliwart zu seinem fïntzigjährigen loktorjubiliam an 6 . August 1914 gewilnet ron Fremden und Schilern (Berlin 1914, Julius Springer), S. 448-451.

Nachweis der Jarstellbarkeit aller gatnzen rationalen Invarianten eines Systems ron Grundformen als ganze alghraische Funktionen gewisser Invarianten.

79. Théorie des corps de nombres algébriques. Exposé, d'apres l'article allemand de D. Milbert (Göttingue), par $H$. Vogt (Tancy). Encyclopédie des sciences mathematiques pures et appliqués Bd. I 3 (Paris und Leipzig 1945, Gauthicr-Villars et Cie. und B. G. Teubner), S. $388-473$.

Mit. Zusätzen versehene Ủbertragung von 57. und 58. ins Französische.

80. Dic Grundlagcn der Physik. Nachrichten von der Königlichen Gesellschaft der Wissenschaften zu Göttingen, mathematisch-physikalische Klasse; Frste Mitteilung, Jahrgang 1915, S. 395-407; /weite Mitteilung, Jahrgang 1917, S. 53-76.

Ableitung der Grundgleichungen der Physik aus den Axiomen von der Weltfunktion und von der allgemeinen Invarianz; Lausalität in der nouen Physik; Geometrie und Physik; Euklidische Pseudogeometrie als Lösung ider physikalischen Grundigleichungen.

s1. Gaston Darboux (1812-191\%). a) Nachrichten von der Königlichen Gesellschaft der Wissenschaíten zu Göttingen, Geschäftliche Mitteilungen, Jahrgang 1917 , S. $71-75$. b) Acta mathematica Bd. 42 (1919), S. 269-273 (Ưbersetzung ins Französis(the).

Gedächtnisrede auf Darboux.

8.). Ariomatisches Denken. a) Mathematische Annilen Bd. 78 (1918), S. 405-415. b) L'enseignement mathématique $\mathrm{Bd}$. 20 (1919), S. $122-136$ (Ubersetzung ins Französische von A. Reymond unter dem Titel: Pensée axiomatique).

Abdruck eines Zürcher Vortrages uber die axiomatische Behandlung der Mathematik.

s3. Adolf Hurwitz. a) Nachrichten von der Königlichen Gesellschaft der Wissenschaften zu Göttingen, Geschäftliche Mitteilungen, Jahrgang 1920, S. $75-83$. b) Mathematische Annalen Bd. 83 (1921), S. $161-172$.

Gedächtnisrede auf Hurwitz. 